\title{
Synthesis and characterization of metal $(\mathrm{M}=\mathrm{Al}$ or $\mathrm{Ga})$ 2-phosphino (phenolate/benzenethiolate) complexes and their electrochemical behavior in the presence of $\mathrm{CO}_{2}$
}

\author{
Jessica A. Bruggen ${ }^{a}$, Keyton D. Feller ${ }^{\mathrm{a}}$, Henry I. Schickel ${ }^{\mathrm{a}}$, Nazym Tuleushova $^{\mathrm{c}}$, \\ Medet Zhukush ${ }^{\mathrm{c}}$, Kevin Lam ${ }^{\mathrm{b}, \mathrm{c}}$, Brian M. Barry ${ }^{\mathrm{a}, *}$ and Richard A. Kemp ${ }^{\mathrm{d}, \mathrm{e}}$ \\ ${ }^{a}$ Department of Chemistry, University of Wisconsin-Platteville, Platteville, WI, USA \\ ${ }^{\mathrm{b}}$ Department of Pharmaceutical, Chemical and Environmental Sciences, Faculty of Engineering and \\ Science, University of Greenwich, Central Avenue, Chatham Maritime, Chatham, Kent, UK \\ ${ }^{\mathrm{c}}$ Department of Chemistry, Nazarbayev University, Astana, Republic of Kazakhstan \\ ${ }^{\mathrm{d}}$ Department of Chemistry and Chemical Biology, University of New Mexico, Albuquerque, NM, USA \\ eAdvanced Materials Laboratory, Sandia National Laboratories, Albuquerque, NM, USA
}

\begin{abstract}
A series of Group 13 complexes $\mathrm{MLX}_{2}\left(\mathrm{M}=\mathrm{Al}\right.$ or $\mathrm{Ga}, \mathrm{L}=\mathrm{SC}_{6} \mathrm{H}_{4}-2-\mathrm{P}^{t} \mathrm{Bu}_{2}$ or $\mathrm{OC}_{6} \mathrm{H}_{4}-2-\mathrm{P}^{t} \mathrm{Bu}_{2}, \mathrm{X}=\mathrm{Me}$ or $\mathrm{C}_{6} \mathrm{~F}_{5}$ ) have been synthesized and characterized by multinuclear NMR spectroscopy and single crystal $\mathrm{X}$-ray diffraction. Reactions of $\mathrm{Me}_{3} \mathrm{Al}$ or $\mathrm{Me}_{3} \mathrm{Ga}$ with an equivalent of either $2-^{t} \mathrm{Bu} 2 \mathrm{P}\left(\mathrm{C}_{6} \mathrm{H}_{4}\right) \mathrm{OH}(\mathbf{1})$ or $2-{ }^{t} \mathrm{Bu}_{2} \mathrm{P}\left(\mathrm{C}_{6} \mathrm{H}_{4}\right) \mathrm{SH}(\mathbf{5})$ resulted in the formation of four new (2,3,6, and 7), 4-coordinate dimethyl chelate (S,P or O,P) complexes via methane elimination. The dimethyl gallium complexes $(3$ and 7$)$ underwent a further reaction with excess $\mathrm{B}\left(\mathrm{C}_{6} \mathrm{~F}_{5}\right)_{3}$, and through ligand exchange (methyl/pentafluorophenyl), resulted in the disubstituted bis(pentafluorophenyl) analogs (4 and 8). Cyclic voltammetry (CV) experiments for all compounds in the presence of and the absence of (1-8) $\mathrm{CO}_{2}$ were performed. For compounds showing cathodic reduction waves under $\mathrm{CO}_{2}(\mathbf{2 , 3 , 4}$, and $\mathbf{6})$, bulk electrolysis experiments were performed. Electrochemical studies indicate that, for several compounds, a transient $\mathrm{CO}_{2}$ adduct is formed which undergoes a one-electron, irreversible (or partially irreversible) reduction to form an unstable radical anion.
\end{abstract}

Keywords: Carbon dioxide, main-group complex, cyclic voltammetry, X-ray crystallography, phosphinophenolate, phosphinobenzenethiolate, frustrated Lewis pair

\section{Introduction}

A mainstay of homogeneous catalysis is the activation of small molecules and their subsequent reactions to afford targeted chemical products, thereby using the small molecule as a building block. One of the most desirable small molecule synthons is $\mathrm{CO}_{2}$, as it is cheap, abundant, and can serve as a renewable source for higher-carbon feedstocks [1]. The activation of $\mathrm{CO}_{2}$ at transition metal centers 
has been thoroughly examined, which has resulted in a variety of metal- $\mathrm{CO}_{2}$ adducts, most often with an electron-rich metal center interacting with the Lewis acidic carbon of $\mathrm{CO}_{2}$ [2]. Far less examined is the activation of $\mathrm{CO}_{2}$ by main-group metal complexes and to an even lesser extent, systems in which a secondary atom in the coordination sphere is involved in the activation of the $\mathrm{CO}_{2}$ [3]. This is somewhat surprising as this is precisely how nature activates $\mathrm{CO}_{2}$ during photosynthesis, a highly effective process for carbon fixation which results in an annual production of $10^{11}$ tons of biomass [ $2 \mathrm{c}$ ].

The coordination of $\mathrm{CO}_{2}$ in an active site of the RuBisCO (Ribulose-1,5-bisphosphate carboxylase/oxygenase) enzyme consists of a dual Lewis acid-base pair. One pair consists of an $\mathrm{O}$ atom from $\mathrm{CO}_{2}$ coordinating to a $\mathrm{Mg}^{2+}$ Lewis acid metal center with a second pair between an $\epsilon-\mathrm{NH}_{2}$ group in lysine and the $\mathrm{C}$ atom in $\mathrm{CO}_{2}$ [4]. It is this evidence that initially inspired our research group to design and synthesize a variety of main-group ( $\mathrm{B}, \mathrm{Ca}, \mathrm{Ge}, \mathrm{In}, \mathrm{Mg}, \mathrm{Pb}, \mathrm{Sr}$ and $\mathrm{Sn}$ ) complexes containing accessible base sites in the ligand architecture, which has resulted in a number of successful dual Lewis acid-base adducts and insertion products upon reaction with $\mathrm{CO}_{2}$ [5]. This synergistic behavior between a Lewis acid (main-group metal) and a Lewis base site (lone-pair base in ligand) towards $\mathrm{CO}_{2}$ complexation has been detailed from the context of frustrated Lewis pairs (FLPs), [6] and there have been several reports of FLPs being used to activate $\mathrm{CO}_{2}$ (See Fig. 1) [3, 7]. With the expansion of FLPs to include Lewis pairs which are not sterically encumbered, [8] one could certainly consider our previous main-group complexes to satisfy the criteria required for FLP status.

Once $\mathrm{CO}_{2}$ is activated, the logical next step is to introduce a secondary reagent targeting chemistry at the $\mathrm{C}$ atom of the $\mathrm{CO}_{2}$, with hopes of releasing new, useful carbon-containing products and regenerating the main-group complex in a catalytic fashion. To this end, several of our previously reported main-group complexes were tested for their behavior in proton-coupled electron transfers (PCETs), where simple, single-carbon products such as $\mathrm{CO}, \mathrm{HCOOH}$, or $\mathrm{CH}_{3} \mathrm{OH}$ were desired [5n, 5o, 9]. Initially, we limited electrochemical experiments to include only complexes persistent enough to reveal crystallographic evidence of $\mathrm{CO}_{2}$ adduct formation, but eventually extended these experiments to various $\mathrm{Zn}$ complexes which demonstrated more fleeting $\mathrm{CO}_{2}$ interactions via FT-IR. One of these complexes $\left[\mathrm{Zn}(2-\mathrm{pyPPh})_{2} \mathrm{Cl}_{2}(\mathrm{py}=\right.$ pyridyl $\left.)\right]$ that exhibited a short-lived $\mathrm{CO}_{2}$ interaction proved to be an effective and robust catalyst for the conversion of $\mathrm{CO}_{2}$ to $\mathrm{CO}$, [9] which is consistent with

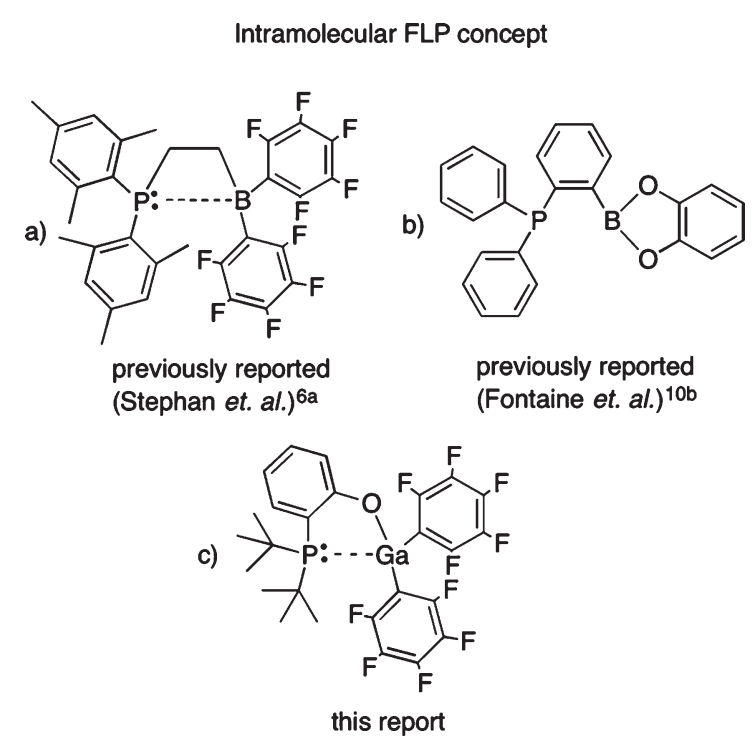

Fig. 1. a) An intramolecular FLP capable of small molecule $\left(\mathrm{CO}_{2} \& \mathrm{H}_{2}\right)$ activation, b) an effective FLP for catalytic conversion of $\mathrm{CO}_{2}$ to $\mathrm{CH}_{3} \mathrm{OH}$ and c) compound 4 from this report. 
other reports that a weak interaction between the catalyst and $\mathrm{CO}_{2}$ is actually a desirable attribute for catalytic $\mathrm{CO}_{2}$ transformations [10]. For example, the compound seen in Fig. 1b (Fontaine et. al.) proved to be an excellent catalyst for the transformation of $\mathrm{CO}_{2}$ to $\mathrm{CH}_{3} \mathrm{OH}$ despite the inability to obtain direct evidence of $\mathrm{CO}_{2}$ interaction with the catalyst. Conversely, the compound seen in Fig. 1a forms a stable, isolable $\mathrm{CO}_{2}$ adduct and no reports of its catalytic abilities have been offered.

Herein, we detail the synthesis and structural characterization of four new dimethyl $\mathrm{Al}$ or $\mathrm{Ga}$ compounds $(\mathbf{2}, \mathbf{3}, \mathbf{6}$, and 7) complexed by either the 2-(di-tert-butylphosphino)benzenethiolate or the 2-(di-tert-butylphosphino)phenolate ligand. The dimethyl Ga complexes were subsequently converted to their bis(pentafluorophenyl) analogs via ligand exchange affording two new complexes ( 4 and 8 ) (See Scheme 1). The design of compounds $\mathbf{4}$ and $\mathbf{8}$ was motivated by the previously reported intramolecular FLPs seen in Fig. 1. As with the compound seen in Fig. 1b, compounds $\mathbf{4}$ and $\mathbf{8}$, offered no direct evidence of $\mathrm{CO}_{2}$ adduct formation via NMR or FT-IR. As such, electrochemical experiments were performed to further probe any possible interactions. Several of the compounds $(\mathbf{2}, \mathbf{3}, \mathbf{4}$, and $\mathbf{6})$ revealed reduction waves in the presence of $\mathrm{CO}_{2}$ while showing no reductions in inert gas environments in the absence of $\mathrm{CO}_{2}$. This direct evidence of a $\mathrm{CE}$ mechanism (chemical reaction followed by electron transfer) indicates that $\mathrm{CO}_{2}$ is in fact interacting with the metal complexes, presumably on a timescale too short-lived for detection via NMR or FT-IR.

\section{Experimental section}

\subsection{Reagents}

The ligands 2- ${ }^{t} \mathrm{Bu}_{2} \mathrm{P}\left(\mathrm{C}_{6} \mathrm{H}_{4}\right) \mathrm{OH}(\mathbf{1})$ and $2-{ }^{t} \mathrm{Bu}_{2} \mathrm{P}\left(\mathrm{C}_{6} \mathrm{H}_{4}\right) \mathrm{SH}(\mathbf{5})$ were prepared as previously reported [5j, 11]. Diethyl ether, pentane and toluene (Aldrich) were further dried using an LC Technology Solutions SP-105 purification system and stored over $4 \AA$ molecular sieves in the glovebox prior to use. The $\mathrm{Me}_{3} \mathrm{Al}, \mathrm{Me}_{3} \mathrm{Ga}$ (Aldrich), and $\mathrm{B}\left(\mathrm{C}_{6} \mathrm{~F}_{5}\right)_{3}$ (TCI) reagents were used as-received without further purification.<smiles>C[M]C(C)(C)C(C)(C)C(C)(C)C</smiles>

$$
\begin{aligned}
& \text { 1: } E=O \\
& \text { 5: } E=S
\end{aligned}
$$

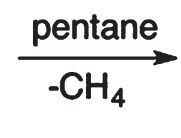

2: $E=O, M=A l, 87 \%$

3: $E=O, M=G a, 67 \%$

6: $E=S, M=A l, 74 \%$

7: $E=S, M=G a, 79 \%$<smiles>CC(C)(C)[Te]P(=Nc1ccccc1F)(C(C)(C)C)C(C)(C)C</smiles>

$\underset{-\mathrm{B}\left(\mathrm{C}_{6} \mathrm{~F}_{5}\right)_{3-\mathrm{x}} \mathrm{Me}_{\mathrm{x}}}{\stackrel{\Delta, \text { toluene }}{\longrightarrow}}$

4: $E=0,32 \%$

8: $E=S, 37 \%$<smiles>CC(C)(C)P1([Ge](C(F)(F)F)(C(F)(F)F)C(F)(F)F)=Cc2ccccc2P1[Ge](C)(C)C(F)(F)F</smiles>

8 and reaction yields. 


\subsection{General methods}

Standard inert atmosphere and Schlenk techniques were used, as all reagents and products were presumed to be air and/or moisture-sensitive. Solution ${ }^{1} \mathrm{H},{ }^{13} \mathrm{C}\left\{{ }^{1} \mathrm{H}\right\},{ }^{31} \mathrm{P}\left\{{ }^{1} \mathrm{H}\right\},{ }^{19} \mathrm{~F}\left\{{ }^{1} \mathrm{H}\right\} \mathrm{NMR}$ spectra were obtained on a Bruker Avance III 300 spectrometer. ${ }^{1} \mathrm{H}$ and ${ }^{13} \mathrm{C}\left\{{ }^{1} \mathrm{H}\right\}$ NMR shifts were referenced to residual solvent peaks downfield of TMS. ${ }^{31} \mathrm{P}\left\{{ }^{1} \mathrm{H}\right\}$ and ${ }^{19} \mathrm{~F}\left\{{ }^{1} \mathrm{H}\right\}$ spectra were referenced to external $85 \% \mathrm{H}_{3} \mathrm{PO}_{4}$, and $\mathrm{CFCl}_{3}$ respectively. Repeated attempts were made to obtain bulk elemental analysis data, but even with added oxidant $\left(\mathrm{Ta}_{2} \mathrm{O}_{5}\right)$ carbon values were always lower than expected, consistent with incomplete combustion [12].

\subsection{Electrochemical methods}

Electrochemical measurements were carried out using an Autolab $302 \mathrm{~N}$ potentiostat interfaced through Nova 2.0 software to a personal computer. Electrochemical measurements were performed using $0.1 \mathrm{M}\left[\mathrm{Bu}_{4} \mathrm{NPF}_{6}\right] / \mathrm{THF}$ electrolyte solutions from solvent that had been purified by passing through an alumina-based purification system. Diamond-polished glassy carbon electrodes of $3 \mathrm{~mm}$ diameter were employed for cyclic voltammetry (CV) scans. CV data were evaluated using standard diagnostic criteria for diffusion control and for chemical and electrochemical reversibility. The experimental reference electrode was a silver wire coated with anodically deposited silver chloride and separated from the working solution by a fine glass frit. The electrochemical potentials in this paper are referenced to ferrocene/ferrocenium couple, as recommended elsewhere [13]. The ferrocene potential was obtained by its addition to the analyte solution at an appropriate time in the experiment $[14]$.

\subsection{Crystallographic studies}

The crystals $(3,4 \&$ 6-8) were coated with Paratone-N oil and were mounted on the nylon fiber of a CryoLoop ${ }^{\mathrm{TM}}$ that had been previously attached to a metallic pin using epoxy. The data were collected at the temperatures indicated in the tables using a Bruker X8 Apex II diffractometer using monochromated Mo K $\alpha$ radiation $(\lambda=0.71073 \AA)$. Data collection and processing were done using the APEX2 software suite [15]. The structures were solved using direct methods and refined with the full matrix least-squares method on F2 with SHELXTL [16]. The nonhydrogen atoms were refined anisotropically. Hydrogen atoms were included at geometrically idealized positions and were not refined. The isotropic thermal parameters of the hydrogen atoms were fixed at $1.5 \mathrm{U}$ equiv. of parent atom for methyl group hydrogens and $1.2 U$ equiv. for all others. All final crystallographic figures shown in this document were generated by Diamond, v. 3.2i [17]. Crystallographic data are given in Table 2.

\subsection{Synthesis of $\left[\mathrm{Me}_{2} \mathrm{Al}\left\{\left(\mathrm{OC}_{6} \mathrm{H}_{4}-2-{ }^{t} \mathrm{Bu} u_{2}\right)-\kappa^{2}-O, P\right\}\right]_{2}$ (2)}

A solution of ${ }^{t} \mathrm{Bu}_{2} \mathrm{P}\left(\mathrm{C}_{6} \mathrm{H}_{4}\right) \mathrm{OH}(0.730 \mathrm{~g}, 3.06 \mathrm{mmol})$ in ca. $15 \mathrm{~mL}$ of pentane is added dropwise over $10 \mathrm{~min}$. to a stirring solution of $\mathrm{Me}_{3} \mathrm{Al}(0.220 \mathrm{~g}, 3.06 \mathrm{mmol})$ in ca. $20 \mathrm{~mL}$ of pentane at $-78{ }^{\circ} \mathrm{C}$. After $30 \mathrm{~min}$. of stirring at $-78{ }^{\circ} \mathrm{C}$, the solution is allowed to warm to room temperature overnight $(15 \mathrm{~h})$, resulting in a clear solution. The volatiles are removed in vacuo resulting in a white powder. The product was purified by recrystallization from $\mathrm{Et}_{2} \mathrm{O}$ at $-18{ }^{\circ} \mathrm{C}$. Yield $=780 \mathrm{mg}(87 \%)$. Colorless, single crystals were grown from $\mathrm{Et}_{2} \mathrm{O}$ at $-18{ }^{\circ} \mathrm{C}$. ${ }^{1} \mathrm{H}$ NMR $\left(\mathrm{CDCl}_{3}, 300 \mathrm{MHz}\right): \delta-0.56(\mathrm{~s}, \mathrm{br}, 6 \mathrm{H}$, $\left.\mathrm{Al}\left(\mathrm{CH}_{3}\right)_{2}\right), 1.34\left(\mathrm{~d},{ }^{3} \mathrm{~J}_{\mathrm{P}-\mathrm{H}}=14 \mathrm{~Hz}, 18 \mathrm{H}, \mathrm{C}\left(\mathrm{CH}_{3}\right)_{3}\right), 6.77\left(\mathrm{~m}, 1 \mathrm{H}, \mathrm{C}_{6} H_{4}\right), 6.92\left(\mathrm{~m}, 1 \mathrm{H}, \mathrm{C}_{6} H_{4}\right), 7.32$ $\left(\mathrm{m}, 1 \mathrm{H}, \mathrm{C}_{6} H_{4}\right), 7.43\left(\mathrm{~m}, 1 \mathrm{H}, \mathrm{C}_{6} H_{4}\right) .{ }^{13} \mathrm{C}\left\{{ }^{1} \mathrm{H}\right\}\left(\mathrm{CDCl}_{3}, 75 \mathrm{MHz}\right): \delta-6.81\left(\mathrm{~s}, \mathrm{br}, \mathrm{Al}\left(\mathrm{CH}_{3}\right)_{2}\right), 29.8$ 
$\left(\mathrm{d},{ }^{2} J_{\mathrm{P}-\mathrm{C}}=6.2 \mathrm{~Hz}, \mathrm{C}\left(\mathrm{CH}_{3}\right)_{3}\right), 33.6\left(\mathrm{~d},{ }^{1} J_{\mathrm{P}-\mathrm{C}}=9.7 \mathrm{~Hz}, C\left(\mathrm{CH}_{3}\right)\right.$, aromatic carbons 118.1, 119.7, 123.3, 133.1. ${ }^{31} \mathrm{P}\left\{{ }^{1} \mathrm{H}\right\}\left(\mathrm{C}_{6} \mathrm{D}_{6}, 121 \mathrm{MHz}\right): \delta 1.09 \mathrm{ppm}$.

\subsection{Synthesis of $\mathrm{Me}_{2} \mathrm{Ga}\left\{\left(\mathrm{OC}_{6} \mathrm{H}_{4}-2-\mathrm{P}^{t} \mathrm{Bu} u_{2}\right)-\kappa^{2}-\mathrm{O}, \mathrm{P}\right\}$ (3)}

A solution of ${ }^{t} \mathrm{Bu}_{2} \mathrm{P}\left(\mathrm{C}_{6} \mathrm{H}_{4}\right) \mathrm{OH}(0.983 \mathrm{~g}, 4.13 \mathrm{mmol})$ in ca. $20 \mathrm{~mL}$ of pentane is added dropwise over $10 \mathrm{~min}$. to a stirring solution of $\mathrm{Me}_{3} \mathrm{Ga}(0.474 \mathrm{~g}, 4.13 \mathrm{mmol})$ in ca. $25 \mathrm{~mL}$ of pentane at $-78{ }^{\circ} \mathrm{C}$. After $30 \mathrm{~min}$. of stirring at $-78{ }^{\circ} \mathrm{C}$, the solution is allowed to warm to room temperature overnight $(15 \mathrm{~h})$, resulting in a clear solution. The volatiles are removed in vacuo resulting in a white powder. The product was purified by recrystallization from $\mathrm{Et}_{2} \mathrm{O}$ at $-18{ }^{\circ} \mathrm{C}$. Yield $=932 \mathrm{mg}(67 \%)$. Colorless, single crystals were grown from $\mathrm{Et}_{2} \mathrm{O}$ at $-18{ }^{\circ} \mathrm{C}$. ${ }^{1} \mathrm{H} \mathrm{NMR}\left(\mathrm{CDCl}_{3}, 300 \mathrm{MHz}\right): \delta-0.08\left(\mathrm{~d},{ }^{3} \mathrm{~J}_{\mathrm{P}-\mathrm{H}}=3.3 \mathrm{~Hz}\right.$, $\left.6 \mathrm{H}, \mathrm{Ga}\left(\mathrm{CH}_{3}\right)_{2}\right), 1.37\left(\mathrm{~d},{ }^{3} J_{\mathrm{P}-\mathrm{H}}=14 \mathrm{~Hz}, 18 \mathrm{H}, \mathrm{C}\left(\mathrm{CH}_{3}\right)_{3}\right), 6.67\left(\mathrm{~m}, 1 \mathrm{H}, \mathrm{C}_{6} H_{4}\right), 6.92\left(\mathrm{~m}, 1 \mathrm{H}, \mathrm{C}_{6} H_{4}\right), 7.30$ $\left(\mathrm{m}, 1 \mathrm{H}, \mathrm{C}_{6} H_{4}\right), 7.41\left(\mathrm{~m}, 1 \mathrm{H}, \mathrm{C}_{6} H_{4}\right) .{ }^{13} \mathrm{C}\left\{{ }^{1} \mathrm{H}\right\}\left(\mathrm{CDCl}_{3}, 75 \mathrm{MHz}\right): \delta-3.50\left(\mathrm{~d},{ }^{2} J_{\mathrm{P}-\mathrm{C}}=19 \mathrm{~Hz}, \mathrm{Ga}\left(\mathrm{CH}_{3}\right)_{2}\right)$, $29.7\left(\mathrm{~d},{ }^{2} J_{\mathrm{P}-\mathrm{C}}=4.7 \mathrm{~Hz}, \mathrm{C}\left(\mathrm{CH}_{3}\right)_{3}\right), 34.4\left(\mathrm{~d},{ }^{1} J_{\mathrm{P}-\mathrm{C}}=15 \mathrm{~Hz}, C\left(\mathrm{CH}_{3}\right)_{3}\right)$, aromatic carbons 115.8, 120.8, 132.6, 133.6, 171.1. ${ }^{31} \mathrm{P}\left\{{ }^{1} \mathrm{H}\right\}\left(\mathrm{C}_{6} \mathrm{D}_{6}, 121 \mathrm{MHz}\right): \delta 9.23 \mathrm{ppm}$.

\subsection{Synthesis of $\left.\left(\mathrm{C}_{6} \mathrm{~F}_{5}\right)_{2} \mathrm{Ga}\left\{\left(\mathrm{OC}_{6} \mathrm{H}_{4}-2-\mathrm{P}^{t} \mathrm{Bu} u_{2}\right)-\kappa^{2}-\mathrm{S}, \mathrm{P}\right]\right\}$ (4)}

A solution of $\mathrm{Me}_{2} \mathrm{Ga}\left\{\left(\mathrm{OC}_{6} \mathrm{H}_{4}-2-\mathrm{P}^{t} \mathrm{Bu}_{2}\right)-\kappa^{2}-\mathrm{O}, \mathrm{P}\right\} \quad(0.441 \mathrm{~g}, 1.31 \mathrm{mmol}), \mathrm{B}\left(\mathrm{C}_{6} \mathrm{~F}_{5}\right)_{3} \quad(1.005 \mathrm{~g}$, $1.96 \mathrm{mmol}$ ) in ca. $35 \mathrm{~mL}$ of toluene is heated to reflux under an $\mathrm{Ar}$ atmosphere for $48 \mathrm{~h}$, resulting in a faint gold-colored translucent solution. The volatiles are removed in vacuo resulting in a sticky, gold-colored solid. The sample is purified by recrystallization from pentane at $-18^{\circ} \mathrm{C}$, resulting in colorless, crystalline product. Yield $=264 \mathrm{mg}(32 \%) .{ }^{1} \mathrm{H} \mathrm{NMR}\left(\mathrm{CDCl}_{3}, 300 \mathrm{MHz}\right): \delta 1.34\left(\mathrm{~d},{ }^{3} J_{\mathrm{P}-\mathrm{H}}=16 \mathrm{~Hz}\right.$, $\left.18 \mathrm{H}, \mathrm{C}\left(\mathrm{CH}_{3}\right)_{3}\right), 6.82\left(\mathrm{~m}, 1 \mathrm{H}, \mathrm{C}_{6} H_{4}\right), 7.14\left(\mathrm{~m}, 1 \mathrm{H}, \mathrm{C}_{6} H_{4}\right), 7.43\left(\mathrm{~m}, 2 \mathrm{H}, \mathrm{C}_{6} H_{4}\right) .{ }^{13} \mathrm{C}\left\{{ }^{1} \mathrm{H}\right\}\left(\mathrm{CDCl}_{3}\right.$, $75 \mathrm{MHz}): \delta 29.2\left(\mathrm{~s}, \mathrm{C}\left(\mathrm{CH}_{3}\right)_{3}\right), 35.6\left(\mathrm{~d},{ }^{1} J_{\mathrm{P}-\mathrm{C}}=19 \mathrm{~Hz}, C\left(\mathrm{CH}_{3}\right)_{3}\right)$, aromatic carbons 117.6, 120.9, 132.4, 134. ${ }^{19} \mathrm{~F}\left\{{ }^{1} \mathrm{H}\right\}\left(\mathrm{C}_{6} \mathrm{D}_{6}, 282 \mathrm{MHz}\right): \delta-160.5\left(\mathrm{~m}, o-\mathrm{C}_{6} F_{5}\right),-151.8\left(\mathrm{t},{ }^{3} J_{\mathrm{F}-\mathrm{F}}=20 \mathrm{~Hz}, p-\mathrm{C}_{6} F_{5}\right),-120.8(\mathrm{~d}$, $\left.{ }^{3} J_{\mathrm{F}-\mathrm{F}}=20 \mathrm{~Hz}, m-\mathrm{C}_{6} F_{5}\right) .{ }^{31} \mathrm{P}\left\{{ }^{1} \mathrm{H}\right\}\left(\mathrm{C}_{6} \mathrm{D}_{6}, 121 \mathrm{MHz}\right): \delta 24.8 \mathrm{ppm}$.

\subsection{Synthesis of $M e_{2} A l\left\{\left(S C_{6} H_{4}-2-P^{t} B u_{2}\right)-\kappa^{2}-S, P\right\}$ (6)}

A solution of ${ }^{t} \mathrm{Bu}_{2} \mathrm{P}\left(\mathrm{C}_{6} \mathrm{H}_{4}\right) \mathrm{SH}(0.553 \mathrm{~g}, 2.17 \mathrm{mmol})$ in ca. $15 \mathrm{~mL}$ of pentane is added dropwise over $10 \mathrm{~min}$. to a stirring solution of $\mathrm{Me}_{3} \mathrm{Al}(0.157 \mathrm{~g}, 2.17 \mathrm{mmol})$ in ca. $20 \mathrm{~mL}$ of pentane at $-78{ }^{\circ} \mathrm{C}$. After $30 \mathrm{~min}$. of stirring at $-78^{\circ} \mathrm{C}$, the solution is allowed to warm to room temperature over $1.5 \mathrm{~h}$, resulting in a clear solution. The volatiles are removed in vacuo resulting in a oily white solid. The crude product is dissolved in a minimal amount of $\mathrm{Et}_{2} \mathrm{O}$, filtered through Celite ${ }^{\circledR}-$ filter aid, and recrystallized at $-18{ }^{\circ} \mathrm{C}$ to give a colorless crystalline product which contained $\mathrm{X}$-ray quality single crystals as well. Yield $=502 \mathrm{mg}(74 \%) .{ }^{1} \mathrm{H} \mathrm{NMR}\left(\mathrm{CDCl}_{3}, 300 \mathrm{MHz}\right): \delta-0.07\left(\mathrm{~d},{ }^{3} \mathrm{~J}_{\mathrm{P}-\mathrm{H}}=2.7 \mathrm{~Hz}, 6 \mathrm{H}, \mathrm{Al}\left(\mathrm{CH}_{3}\right)_{2}\right), 1.03(\mathrm{~d}$, $\left.{ }^{3} J_{\mathrm{P}-\mathrm{H}}=14 \mathrm{~Hz}, 18 \mathrm{H}, \mathrm{C}\left(\mathrm{CH}_{3}\right)_{3}\right), 6.71\left(\mathrm{~m}, 1 \mathrm{H}, \mathrm{C}_{6} H_{4}\right), 6.89\left(\mathrm{~m}, 1 \mathrm{H}, \mathrm{C}_{6} H_{4}\right), 7.13\left(\mathrm{~m}, 1 \mathrm{H}, \mathrm{C}_{6} H_{4}\right), 7.81(\mathrm{~m}$, $\left.1 \mathrm{H}, \mathrm{C}_{6} H_{4}\right) .{ }^{13} \mathrm{C}\left\{{ }^{1} \mathrm{H}\right\}\left(\mathrm{CDCl}_{3}, 75 \mathrm{MHz}\right): \delta-5.80\left(\mathrm{~s}, \mathrm{br}, \mathrm{Al}\left(\mathrm{CH}_{3}\right)_{2}\right), 29.2\left(\mathrm{~d},{ }^{2} \mathrm{~J}_{\mathrm{P}-\mathrm{C}}=4.4 \mathrm{~Hz}, \mathrm{C}\left(\mathrm{CH}_{3}\right)_{3}\right)$, $34.8\left(\mathrm{~d},{ }^{1} J_{\mathrm{P}-\mathrm{C}}=13 \mathrm{~Hz}, C\left(\mathrm{CH}_{3}\right)\right.$, aromatic carbons $122.4,131.3,133.5,134.0,154.6 .{ }^{31} \mathrm{P}\left\{{ }^{1} \mathrm{H}\right\}\left(\mathrm{C}_{6} \mathrm{D}_{6}\right.$, $121 \mathrm{MHz}): \delta 21.9 \mathrm{ppm}$.

\subsection{Synthesis of $\mathrm{Me}_{2} \mathrm{Ga}\left\{\left(\mathrm{SC}_{6} \mathrm{H}_{4}-2-\mathrm{P}^{t} \mathrm{Bu} 2\right)-\kappa^{2}-S, P\right\}$ (7)}

A solution of ${ }^{t} \mathrm{Bu}_{2} \mathrm{P}\left(\mathrm{C}_{6} \mathrm{H}_{4}\right) \mathrm{SH}(0.420 \mathrm{~g}, 1.65 \mathrm{mmol})$ in ca. $15 \mathrm{~mL}$ of pentane is added dropwise over $10 \mathrm{~min}$. to a stirring solution of $\mathrm{Me}_{3} \mathrm{Ga}(0.190 \mathrm{~g}, 1.65 \mathrm{mmol})$ in ca. $20 \mathrm{~mL}$ of pentane at $-78{ }^{\circ} \mathrm{C}$. After $30 \mathrm{~min}$. of stirring at $-78^{\circ} \mathrm{C}$, the solution is allowed to warm to room temperature over $1.5 \mathrm{~h}$, resulting in a clear solution. The volatiles are removed in vacuo resulting in a white solid. Yield $=458 \mathrm{mg}$ 
(79\%). Colorless, single crystals were grown from $\mathrm{Et}_{2} \mathrm{O}$ at $-18{ }^{\circ} \mathrm{C} .{ }^{1} \mathrm{H} \mathrm{NMR}\left(\mathrm{CDCl}_{3}, 300 \mathrm{MHz}\right): \delta$ $0.28\left(\mathrm{~d},{ }^{3} J_{\mathrm{P}-\mathrm{H}}=3.6 \mathrm{~Hz}, 6 \mathrm{H}, \mathrm{Ga}\left(\mathrm{CH}_{3}\right)_{2}\right), 1.02\left(\mathrm{~d},{ }^{3} J_{\mathrm{P}-\mathrm{H}}=14 \mathrm{~Hz}, 18 \mathrm{H}, \mathrm{C}\left(\mathrm{CH}_{3}\right)_{3}\right), 6.69\left(\mathrm{~m}, 1 \mathrm{H}, \mathrm{C}_{6} H_{4}\right)$, $6.89\left(\mathrm{~m}, 1 \mathrm{H}, \mathrm{C}_{6} H_{4}\right), 7.18\left(\mathrm{~m}, 1 \mathrm{H}, \mathrm{C}_{6} H_{4}\right), 7.84\left(\mathrm{~m}, 1 \mathrm{H}, \mathrm{C}_{6} H_{4}\right) .{ }^{13} \mathrm{C}\left\{{ }^{1} \mathrm{H}\right\}\left(\mathrm{CDCl}_{3}, 75 \mathrm{MHz}\right): \delta-2.91$ (s, br, $\left.\mathrm{Ga}\left(\mathrm{CH}_{3}\right)_{2}\right), 28.6\left(\mathrm{~d},{ }^{2} \mathrm{~J}_{\mathrm{P}-\mathrm{C}}=4.2 \mathrm{~Hz}, \mathrm{C}\left(\mathrm{CH}_{3}\right)_{3}\right), 34.7\left(\mathrm{~d},{ }^{1} \mathrm{~J}_{\mathrm{P}-\mathrm{C}}=14 \mathrm{~Hz}, \mathrm{C}\left(\mathrm{CH}_{3}\right)\right.$, aromatic carbons $120.8,130.5,132.8,133.2 .{ }^{31} \mathrm{P}\left\{{ }^{1} \mathrm{H}\right\}\left(\mathrm{C}_{6} \mathrm{D}_{6}, 121 \mathrm{MHz}\right): \delta 32.4 \mathrm{ppm}$.

\subsection{Synthesis of $\left(\mathrm{C}_{6} \mathrm{~F}_{5}\right)_{2} \mathrm{Ga}\left\{\left(\mathrm{SC}_{6} \mathrm{H}_{4}-2-\mathrm{P}^{t} \mathrm{Bu} \mathrm{u}_{2}\right)-\kappa^{2}-\mathrm{S}, \mathrm{P}\right\}$ (8)}

A solution of $3(0.302 \mathrm{~g}, 0.86 \mathrm{mmol}), \mathrm{B}\left(\mathrm{C}_{6} \mathrm{~F}_{5}\right)_{3}(0.657 \mathrm{~g}, 1.28 \mathrm{mmol})$ in ca. $40 \mathrm{~mL}$ of toluene is heated to reflux under an argon atmosphere for $48 \mathrm{~h}$, resulting in a faint gold-colored translucent solution. The volatiles are removed in vacuo resulting in a sticky, gold-colored solid. The sample is purified by recrystallization from pentane at $-18{ }^{\circ} \mathrm{C}$, resulting in colorless, crystalline product. Yield $=207 \mathrm{mg}$ (37\%). ${ }^{1} \mathrm{H} \mathrm{NMR}\left(\mathrm{CDCl}_{3}, 300 \mathrm{MHz}\right): \delta 0.94\left(\mathrm{~d},{ }^{3} J_{\mathrm{P}-\mathrm{H}}=15 \mathrm{~Hz}, 18 \mathrm{H}, \mathrm{C}\left(\mathrm{CH}_{3}\right)_{3}\right), 6.69\left(\mathrm{~m}, 1 \mathrm{H}, \mathrm{C}_{6} H_{4}\right)$, $6.90\left(\mathrm{~m}, 1 \mathrm{H}, \mathrm{C}_{6} H_{4}\right), 7.07\left(\mathrm{~m}, 1 \mathrm{H}, \mathrm{C}_{6} H_{4}\right), 7.77\left(\mathrm{~m}, 1 \mathrm{H}, \mathrm{C}_{6} H_{4}\right) .{ }^{13} \mathrm{C}\left\{{ }^{1} \mathrm{H}\right\}\left(\mathrm{CDCl}_{3}, 75 \mathrm{MHz}\right): \delta 29.0(\mathrm{~s}$, $\left.\mathrm{C}\left(\mathrm{CH}_{3}\right)_{3}\right), 36.6\left(\mathrm{~d},{ }^{1} J_{\mathrm{P}-\mathrm{C}}=15 \mathrm{~Hz}, C\left(\mathrm{CH}_{3}\right)_{3}\right)$, aromatic carbons $123.5,123.7,132.2,133.5 .{ }^{19} \mathrm{~F}\left\{{ }^{1} \mathrm{H}\right\}$ $\left(\mathrm{C}_{6} \mathrm{D}_{6}, 282 \mathrm{MHz}\right): \delta-160.7\left(\mathrm{~m}, m-\mathrm{C}_{6} F_{5}\right),-152.3\left(\mathrm{t},{ }^{3} J_{\mathrm{F}-\mathrm{F}}=20 \mathrm{~Hz}, p-\mathrm{C}_{6} F_{5}\right),-118.7\left(\mathrm{~d},{ }^{3} J_{\mathrm{F}-\mathrm{F}}=20 \mathrm{~Hz}\right.$, $\left.o-\mathrm{C}_{6} F_{5}\right) .{ }^{31} \mathrm{P}\left\{{ }^{1} \mathrm{H}\right\}\left(\mathrm{C}_{6} \mathrm{D}_{6}, 121 \mathrm{MHz}\right): \delta 32.5 \mathrm{ppm}$.

\section{Results and discussion}

\subsection{Preparation and characterization}

The 2- ${ }^{t} \mathrm{Bu}_{2} \mathrm{P}\left(\mathrm{C}_{6} \mathrm{H}_{4}\right) \mathrm{OH}(\mathbf{1})$ and $2-{ }^{t} \mathrm{Bu}_{2} \mathrm{P}\left(\mathrm{C}_{6} \mathrm{H}_{4}\right) \mathrm{SH}(\mathbf{5})$ proligands were synthesized according to literature methods, $[5 \mathrm{j}, 11]$ and were diluted in pentane before addition to solutions of either $\mathrm{Me}_{3} \mathrm{Ga}$ or $\mathrm{Me}_{3} \mathrm{Al}$, which were also diluted in pentane. Furthermore, prior to addition the reaction flasks were cooled to $-78^{\circ} \mathrm{C}$ in an ${ }^{i} \mathrm{PrOH}$ slurry cooled by $\mathrm{N}_{2(l)}$. These precautions were taken in attempts to avoid the disubstitution of the metal center and to maximize production of the monoligated products $\mathrm{Me}_{2} \mathrm{ML}$ (compounds $2,3,6$, and 7). After warming to room temperature (15 h), the solvent was removed in vacuo and products were then purified via recrystallization from saturated $\mathrm{Et}_{2} \mathrm{O}$ solutions at $-18{ }^{\circ} \mathrm{C}$, resulting in colorless crystalline material in respectable yields $(>65 \%)$ in each instance. It should also be noted that for these four compounds, X-ray quality crystals were also obtained from concentrated $\mathrm{Et}_{2} \mathrm{O}$ solutions at $-18{ }^{\circ} \mathrm{C}$ (Table 1$)$.

Table 1

Select bond lengths, angles, chemical shifts and J coupling constants

\begin{tabular}{lcccccccc}
\hline & $\mathrm{M}^{a}-\mathrm{P}(\AA)$ & $\begin{array}{c}\mathrm{M}-\mathrm{E}^{b} \\
\AA\end{array}$ & $\begin{array}{c}\angle \mathrm{E}-\mathrm{M}-\mathrm{P} \\
(\mathrm{A})\end{array}$ & $\delta{ }^{31} \mathrm{P}^{c}$ & $\begin{array}{c}\delta{ }^{13} \mathrm{C}\left({ }^{1} J_{\mathrm{P}-\mathrm{C}}\right)^{d} \\
C\left(\mathrm{CH}_{3}\right)_{3}\end{array}$ & $\begin{array}{c}\delta{ }^{13} \mathrm{C}\left({ }^{2} J_{\mathrm{P}-\mathrm{C}}\right)^{d} \\
\mathrm{C}\left(\underline{C}_{3}\right)_{3}\end{array}$ & $\begin{array}{c}\delta{ }^{13} \mathrm{C}^{d} \\
\mathrm{M}\left(\underline{C} \mathrm{H}_{3}\right)_{2}\end{array}$ & $\begin{array}{c}\delta{ }^{1} \mathrm{H}^{d} \\
\mathrm{M}\left(\mathrm{C} \underline{H}_{3}\right)_{2}\end{array}$ \\
\hline $\mathbf{2}$ & - & - & - & 1.09 & $33.6(9.7)$ & $29.8(6.2)$ & -6.81 & -0.56 \\
$\mathbf{3}$ & $2.4427(3)$ & $1.9119(9)$ & $85.22(3)$ & 9.23 & $34.4(15)$ & $29.7(4.7)$ & -3.50 & -0.08 \\
$\mathbf{4}$ & $2.3939(10)$ & $1.868(2)$ & $86.79(8)$ & 24.9 & $35.6(19)$ & $29.2(-)^{e}$ & - & - \\
$\mathbf{6}$ & $2.4825(8)$ & $2.2865(8)$ & $87.60(3)$ & 21.9 & $34.8(13)$ & $29.2(4.4)$ & -5.80 & -0.07 \\
$\mathbf{7}$ & $2.4509(6)$ & $2.3203(7)$ & $87.83(2)$ & 32.4 & $34.7(14)$ & $28.6(4.2)$ & -2.91 & 0.28 \\
$\mathbf{8}$ & $2.4080(7)$ & $2.2736(7)$ & $90.04(2)$ & 32.5 & $36.6(15)$ & $29.0(-)^{e}$ & - & - \\
\hline
\end{tabular}

${ }^{a} \mathrm{M}=\mathrm{Al}\left(2\right.$ \& 6), $\mathrm{Ga}\left(\mathbf{3}, \mathbf{4}, 7\right.$ \& 8), ${ }^{b} \mathrm{E}=\mathrm{O}(\mathbf{2}-\mathbf{4}), \mathrm{S}(\mathbf{6}-\mathbf{8}),{ }^{c} \mathrm{All}{ }^{31} \mathrm{P}$ performed in $\mathrm{C}_{6} \mathrm{D}_{6}$ shifts reported in ppm, ${ }^{d} \mathrm{All}{ }^{13} \mathrm{C}$ and ${ }^{1} \mathrm{H}$ performed in $\mathrm{CDCl}_{3}$, all shifts reported in ppm and $J$ coupling constants reported in $\mathrm{Hz},{ }^{e} \mathrm{These}$ signals were slightly broad and the ${ }^{2} J_{\mathrm{P}-\mathrm{C}}$ coupling constants could not be resolved. 


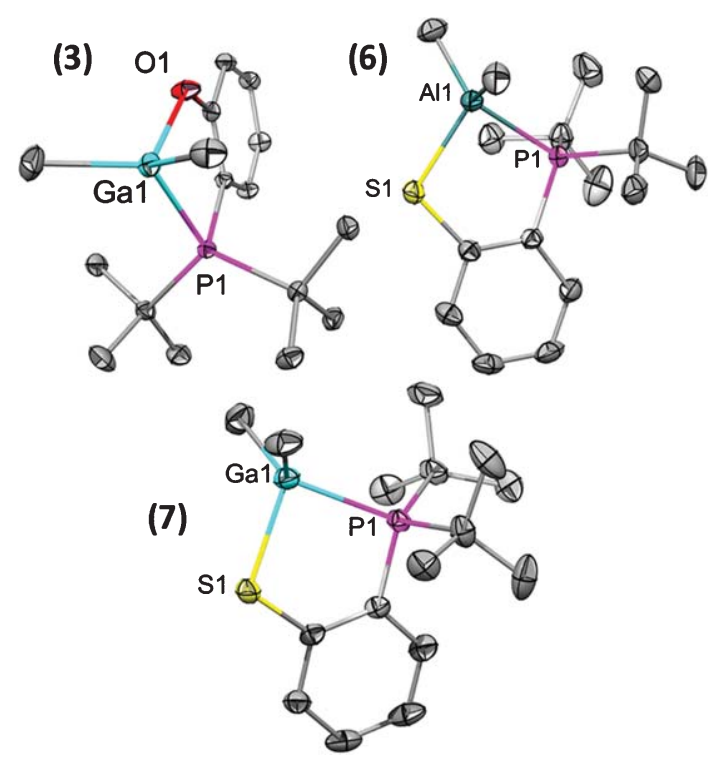

Fig. 2. Molecular structures of 3, 6, and 7 (50\% ellipsoids). Hydrogens have been omitted for clarity and carbon atoms are shown in grey. Select bond lengths and angles can be seen in Table 1.

The transformation of the dimethyl Ga complexes $\mathbf{3}$ and $\mathbf{7}$ to their bis(pentafluorophenyl) analogs was then targeted and was achieved by heating $\mathbf{3}$ and $\mathbf{7}$ in refluxing toluene overnight with an excess of $\mathrm{B}\left(\mathrm{C}_{6} \mathrm{~F}_{5}\right)_{3}$. The concept of $\mathrm{Me} / \mathrm{C}_{6} \mathrm{~F}_{5}$ ligand exchange was first introduced by Klosin et al. where it was shown that the reaction of $\mathrm{B}\left(\mathrm{C}_{6} \mathrm{~F}_{5}\right)_{3}$ with $\mathrm{Me}_{3} \mathrm{Al}$ produced $\mathrm{Al}\left(\mathrm{C}_{6} \mathrm{~F}_{5}\right)_{3}$ and $\mathrm{Me}_{3} \mathrm{~B}$ when reacted in non-coordinating solvents [18]. When adopting this methodology, initial attempts were performed adding enough $\mathrm{B}\left(\mathrm{C}_{6} \mathrm{~F}_{5}\right)_{3}$ such that the methyl and pentafluorophenyl groups were in a 1:1 stoichiometric ratio, or 0.66 equivalents of $\mathrm{B}\left(\mathrm{C}_{6} \mathrm{~F}_{5}\right)_{3}$ per metal center. This resulted in an incomplete exchange as evidence by isolated crystals of a monosubstituted complex $\operatorname{LGaMe}\left(\mathrm{C}_{6} \mathrm{~F}_{5}\right)$ (where $\left.\mathrm{L}=\mathrm{SC}_{6} \mathrm{H}_{4}-2-\mathrm{P}^{t} \mathrm{Bu}_{2}\right)$. When the amount of $\mathrm{B}\left(\mathrm{C}_{6} \mathrm{~F}_{5}\right)_{3}$ was increased to 1.5 equivalents per metal center, the bis(pentafluorophenyl) analogs $\mathbf{4}$ and $\mathbf{8}$ were successfully produced along with non-volatile $\mathrm{B}\left(\mathrm{C}_{6} \mathrm{~F}_{5}\right)_{3-\mathrm{x}} \mathrm{Me}_{\mathrm{x}}$ byproducts. The crude mixture of products after solvent removal was a sticky solid, but the pure products could be isolated via recrystallization from pentane at $-18^{\circ}$, albeit in lowered yields $(\sim 30 \%)$.

Multinuclear NMR spectra were collected for species 2-4 and 6-8, resulting in singlets in the ${ }^{31} \mathrm{P}$ experiments for all compounds with chemical shifts ranging from $\sim 1-33$ ppm (see Table 1) when measured in $\mathrm{C}_{6} \mathrm{D}_{6}$. These results indicate that the ligand, at least at room temperature, is not exhibiting any hemilability towards the metal center. We have reported previously an instance when the $2-{ }^{t} \mathrm{Bu}_{2} \mathrm{P}-$ $\mathrm{C}_{6} \mathrm{H}_{4} \mathrm{~S}^{-}$ligand does exhibit room temperature hemilabile behavior in a $\mathrm{Sn}^{\mathrm{IV}}$ complex [5j]. The presence of two signals in the ${ }^{31} \mathrm{P}$ NMR spectrum which have temperature-dependent relative integration ratios, along with Sn-P coupling constant data, clearly reveal the hemilabile behavior. The lack of hemilabile behavior in the compounds reported here is not completely surprising due to the lack of ring strain in the resulting 5-membered chelate rings and with the strong Lewis acid nature of $\mathrm{Al}^{3+}$ and $\mathrm{Ga}^{3+}$ metal centers. When comparing the chemical shifts of compound $\mathbf{2}$ to those in compound $\mathbf{3}$ as well as $\mathbf{6}$ to 7, the only difference is the identity of the metal center. In both pairs of comparisons, it is clear by looking at chemical shifts from ${ }^{31} \mathrm{P}$ and ${ }^{13} \mathrm{C}$ NMR spectra of atoms directly bound to the metal center ( $\mathrm{P}$ and $\mathrm{C}$ ), that the Ga center is deshielding its nearest neighbors noticeably more than the $\mathrm{Al}$ analogs. For example, the ${ }^{31} \mathrm{P}$ signal is $1.09 \mathrm{ppm}$ for $\mathbf{2}$ while compound $\mathbf{3}$ is further downfield at $9.23 \mathrm{ppm}$. This trend is also seen when looking at the ${ }^{13} \mathrm{C}\left\{{ }^{1} \mathrm{H}\right\}$ and ${ }^{1} \mathrm{H}$ NMR of the metal-bound methyl groups (see 


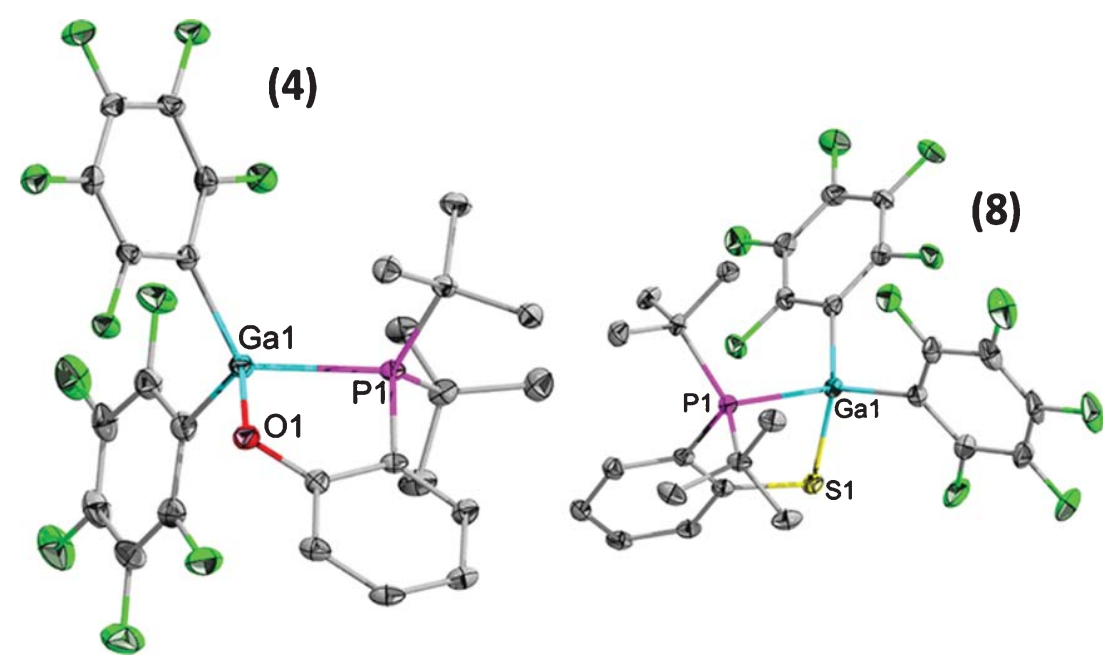

Fig. 3. Molecular structures of $\mathbf{4}$ and $\mathbf{8}$ (50\% ellipsoids). Hydrogens have been omitted for clarity, carbon atoms are shown in grey, and fluorine atoms in green. Select bond lengths and angles can be seen in Table 1.

Table 1). In another set of comparisons, the effect that the chalcogenide identity (E) has on chemical shifts can be scrutinized by comparing pairs of compounds 2 to 6 and 3 to 7. In both instances, the $S$ containing compounds $(6 \& 7)$ revealed a downfield shift in the metal-bound atoms relative to their phenolate analogs ( $2 \& 3$ ) (see Table 1 ). For example, the ${ }^{31} \mathrm{P}$ signal in compound $\mathbf{3}$ is at $9.23 \mathrm{ppm}$ while in compound 7 it is at 32.4 ppm.

Crystal structures were collected for compounds $3,4,6,7$, and 8 revealing distorted tetrahedral metal center geometries for all compounds (see Figs. 2 and 3). Crystallographic data and parameters for these compounds has been complied in Table 2. Exhaustive attempts were made to obtain a high quality solution for X-ray data collected for compound 2, but these were not successful. However, although we obtained poor data, it was clear from our efforts to solve the crystal structure of 2 that it dimerizes in the solid state resulting in a 5-coordinate distorted trigonal bipyramidal coordination environment around the Al. The dimerization occurs through adduct formation between an $\mathrm{O}$ lone pair from the phenolate coordinating to a neighboring Al. The equatorial positions in $\mathbf{2}$ are occupied by two methyl groups and the covalently bound $\mathrm{O}$ atom from the $2{ }_{-}{ }^{t} \mathrm{Bu}_{2} \mathrm{P}_{-} \mathrm{C}_{6} \mathrm{H}_{4} \mathrm{O}^{-}$ligand while the axial positions are occupied by the $\mathrm{P}$ atom from the same $2{ }^{-}{ }^{t} \mathrm{Bu}_{2} \mathrm{P}_{-} \mathrm{C}_{6} \mathrm{H}_{4} \mathrm{O}^{-}$ligand and the $\mathrm{O}$ atom from a neighboring molecule. Aside from the dimerization of compound $\mathbf{2}$, there are no remarkable or unexpected crystallographic features found in the structures seen in Figs. 2 and 3. For the bis(pentafluorophenyl) compounds $\mathbf{4}$ and $\mathbf{8}$, the expectation is that the strong electron withdrawing nature of the perfluorinated substituents will shorten the Ga-P and Ga-E (E=O or $\mathrm{S}$ ) bonds relative to their dimethyl counterparts. This expectation holds true as the Ga-P bond lengths and Ga-E bond lengths for compounds $\mathbf{4}$ and $\mathbf{8}$ are $\sim 0.05 \AA$ shorter than of those found in compounds $\mathbf{3}$ and $\mathbf{7}$ (see Table 1).

\section{2. $\mathrm{CO}_{2}$ reactivity studies}

Upon successful isolation and characterization of compounds $2,3,4,6,7$, and 8 , focus was then turned to probing the reactivity of these compounds with $\mathrm{CO}_{2}$. Initial experiments looked for evidence of $\mathrm{CO}_{2}$ complex interactions via FT-IR and NMR after bubbling $\mathrm{CO}_{2}$ through solutions of the aforementioned compounds. If stable adducts were to form, there would be clear evidence of this in both the NMR and FT-IR spectra when compared to the complexes before exposure to $\mathrm{CO}_{2}$. For example, in one of our 
Table 2

Crystallographic Data and Parameters for Compounds 3, 4, \& 6-8

\begin{tabular}{|c|c|c|c|c|c|}
\hline & 3 & 4 & 6 & 7 & 8 \\
\hline empirical formula & $\mathrm{C}_{16} \mathrm{H}_{28} \mathrm{GaOP}$ & $\mathrm{C}_{26} \mathrm{H}_{22} \mathrm{~F}_{10} \mathrm{GaOP}$ & $\mathrm{C}_{16} \mathrm{H}_{28}$ AlPS & $\mathrm{C}_{16} \mathrm{H}_{28} \mathrm{GaPS}$ & $\mathrm{C}_{26} \mathrm{H}_{22} \mathrm{~F}_{10} \mathrm{GaPS}$ \\
\hline FW & 337.07 & 641.13 & 310.39 & 353.13 & 659.20 \\
\hline$T, \mathrm{~K}$ & $173(2)$ & $173(2)$ & $173(2)$ & $173(2)$ & $173(2)$ \\
\hline cryst size $(\mathrm{mm})$ & $0.50 \times 0.50 \times 0.19$ & $0.34 \times 0.26 \times 0.12$ & $0.58 \times 0.30 \times 0.22$ & $0.46 \times 0.34 \times 0.30$ & $0.37 \times 0.34 \times 0.34$ \\
\hline cryst syst & monoclinic & monoclinic & orthorhombic & orthorhombic & monoclinic \\
\hline space group & $\mathrm{P} 2(1) / c$ & $\mathrm{P} 2(1) / n$ & Pbca & Pbca & $\mathrm{P} 2(1) / n$ \\
\hline$a, \AA$ & $13.9275(14)$ & $9.9121(4)$ & $14.0758(5)$ & $14.0468(6)$ & $8.6475(2)$ \\
\hline$b, \AA$ & $9.6095(9)$ & $11.3953(4)$ & $15.4382(6)$ & $15.4442(6)$ & $25.4338(7)$ \\
\hline$c, \AA$ & $12.9652(13)$ & $23.0044(9)$ & $16.5577(6)$ & $16.5316(7)$ & $12.4720(3)$ \\
\hline$\alpha, \operatorname{deg}$ & 90.00 & 90.00 & 90.00 & 90.00 & 90.00 \\
\hline$\beta, \operatorname{deg}$ & $96.635(4)$ & $95.846(2)$ & 90.00 & 90.00 & $91.6240(10)$ \\
\hline$\gamma, \operatorname{deg}$ & 90.00 & 90.00 & 90.00 & 90.00 & 90.00 \\
\hline vol, $\AA^{3}$ & $1723.6(3)$ & $2584.86(17)$ & $3598.1(2)$ & $3586.4(3)$ & $2741.97(12)$ \\
\hline$Z$ & 4 & 4 & 8 & 8 & 4 \\
\hline calcd density $\left(\mathrm{g} / \mathrm{cm}^{3}\right)$ & 1.299 & 1.647 & 1.146 & 1.308 & 1.597 \\
\hline$\mu, \mathrm{mm}^{-1}$ & 1.681 & 1.218 & 0.305 & 1.727 & 1.221 \\
\hline $\mathrm{R} 1[\mathrm{I}>2 \sigma(I)]^{a}$ & 0.0204 & 0.0395 & 0.0351 & 0.0285 & 0.0333 \\
\hline $\mathrm{wR} 2[\mathrm{I}>2 \sigma(I)]^{b}$ & 0.0558 & 0.0999 & 0.0981 & 0.0896 & 0.0901 \\
\hline
\end{tabular}

${ }^{a} \mathrm{R} 1=\Sigma F_{o}|-| F_{c} / \Sigma\left|F_{o}\right| \cdot{ }^{b} \mathrm{wR} 2=\left\{\Sigma\left[w\left(F_{o}{ }^{2}-F_{c}{ }^{2}\right)^{2}\right] / \Sigma\left[w\left(F_{o}{ }^{2}\right)^{2}\right]\right\}^{1 / 2}$.

previous studies, a P,P-chelated stannylene $\left[\left({ }^{i} \mathrm{Pr}_{2} \mathrm{P}\right)_{2} \mathrm{~N}\right]_{2} \mathrm{Sn}$ formed an isolable $\mathrm{CO}_{2}$ dual Lewis acidbase adduct upon insertion of $\mathrm{CO}_{2}$ into one of the $\mathrm{Sn}-\mathrm{P}$ bonds [5c]. Evidence for $\mathrm{CO}_{2}$ incorporation was clearly demonstrated by both FT-IR and NMR as a strong $\mathrm{C}=\mathrm{O}$ absorbance at $1629 \mathrm{~cm}^{-1}$ was seen and the chemical resonance of the $\mathrm{P}$ atom directly bound to the $\mathrm{C}$ in $\mathrm{CO}_{2}$ could be observed.

In another example, [9] our group synthesized a $\mathrm{Zn}$ complex $\left(\left[\mathrm{Ph}_{2} \mathrm{Ppy}\right]_{2} \mathrm{ZnCl}_{2}\right.$, py $=2$-pyridyl $)$ which showed no evidence of $\mathrm{CO}_{2}$ interaction via NMR, even when experiments were performed at $100 \mathrm{psig}$ $\mathrm{CO}_{2}$ and at $-48^{\circ} \mathrm{C}$. However, a short-lived ( $\sim 10$ minutes $) \mathrm{C}=\mathrm{O}$ stretch could be observed at $1726 \mathrm{~cm}^{-1}$ in the solid state FT-IR spectrum. The higher wavenumber seen in the Zn complex is consistent with a weaker, more transient $\mathrm{CO}_{2}$-complex interaction, and is consistent with the inability to isolate a stable adduct or observe any change in the NMR spectra upon $\mathrm{CO}_{2}$ exposure. The $\mathrm{Zn}$ complex did however prove to be a robust electrocatalyst for the reduction of $\mathrm{CO}_{2}$ to $\mathrm{CO}$, whereas the $\mathrm{Sn}-\mathrm{CO}_{2}$ complex was not, suggesting that perhaps a weaker interaction between $\mathrm{CO}_{2}$ and the complex is desirable for catalytic transformations of the $\mathrm{CO}_{2}$.

After exposing compounds $\mathbf{2}, \mathbf{3}, \mathbf{4}, \mathbf{6}, \mathbf{7}$, and $\mathbf{8}$ to $\mathrm{CO}_{2}$, no evidence of an adduct could be observed via NMR or FT-IR even when elevated pressures $(10 \mathrm{~atm})$ and extended exposure durations $(15 \mathrm{~h})$ were employed. This lack of evidence, however, did not deter further examination of these compounds for their ability to activate $\mathrm{CO}_{2}$ as we have stated that other reports have revealed that a complex (See Fig. 1b) that lacks evidence of $\mathrm{CO}_{2}$ interaction via NMR and FT-IR is still capable of performing as a catalyst for $\mathrm{CO}_{2}$ transformations [10b]. In this study, it was revealed that $\mathrm{CO}_{2}$ was only susceptible to chemical reduction in the presence of catalyst providing indirect evidence of $\mathrm{CO}_{2}$ activation. In contrast to this report, we aim to use electrochemical reduction to provide direct evidence of $\mathrm{CO}_{2}$ activation by the $\mathrm{Al}$ and $\mathrm{Ga}$ complexes $2,3,4,6,7$, and 8 . Evidence for a $\mathrm{CE}$ mechanism, which in this case establishes $\mathrm{CO}_{2}$ activation, can be clearly demonstrated if the reduction onset potential of the complex in the presence of $\mathrm{CO}_{2}$ is lowered relative to the onset potential of the complexes in an inert gas environment. 

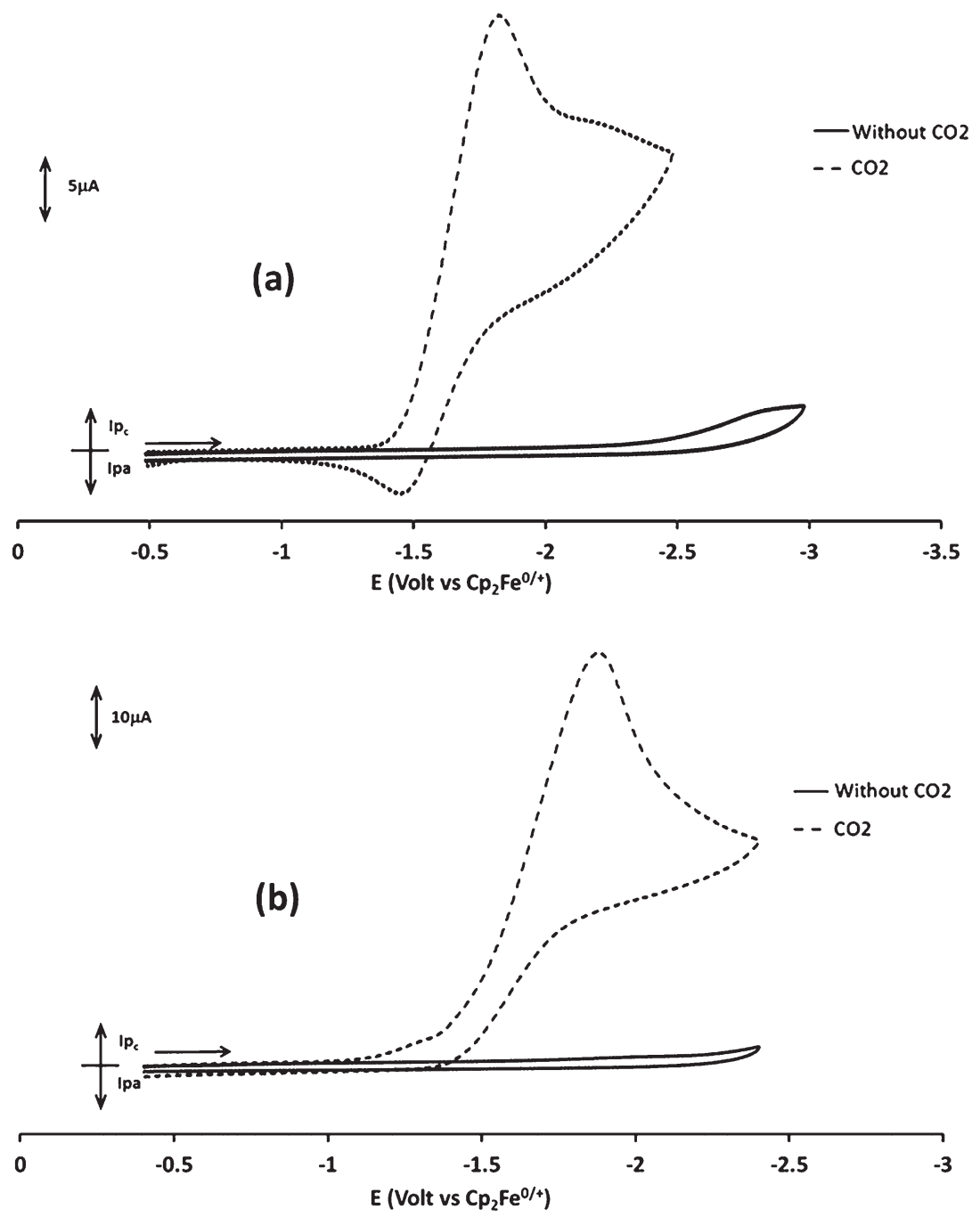

Fig. 4. Cyclic Voltammetry scans of (a) $1 \mathrm{mM} 3$ and (b) $1 \mathrm{mM} 4$ both in THF/0.1 M $\left[\mathrm{NBu}_{4}\right]\left[\mathrm{PF}_{6}\right]$ at $3 \mathrm{~mm}$ glassy carbon electrodes, and both with scan rates of $0.2 \mathrm{~V} \mathrm{~s}^{-1}$.

\subsection{Electrochemical studies}

The electrochemical properties of all metal complexes were investigated using CV experiments, and four of the six compounds demonstrated cathodic waves consistent with a CE mechanism when exposed to $\mathrm{CO}_{2}$. Compounds 7 and $\mathbf{8}$ showed a complex series of reduction in absence of $\mathrm{CO}_{2}$. No difference in their cathodic behavior was observed when the compounds were exposed to $\mathrm{CO}_{2}$, and as such will not be discussed further in this section. Complexes $\mathbf{2 , 3 , 4}$, and $\mathbf{6}$, however, display very similar cathodic behaviors. In the absence of $\mathrm{CO}_{2}$, none of the compounds showed any reduction waves within the limits of the solvent window. However, when $\mathrm{CO}_{2}$ was passed through the electrochemical cell, a diffusion-controlled electrochemically-reversible but chemically-partially reversible cathodic reduction was observed at the potentials collected in Table 3 for $\mathbf{2 , 3}, \mathbf{4}$, and $\mathbf{6}$. Overlayed CV scans of these four compounds in the presence of and in the absence of $\mathrm{CO}_{2}$ is shown in Fig. 4 for compounds 3 and $\mathbf{4}$, and in the supporting information (Figure S3) for $\mathbf{2}$ and 6. Compound $\mathbf{4}$ was the only species that displayed a diffusion-controlled both electrochemically and chemically-irreversible cathodic reduction [19]. Voltammetry at higher sweeping rates (up to $250,000 \mathrm{~V} \cdot \mathrm{s}^{-1}$ ) and at low temperature $\left(-50^{\circ} \mathrm{C}\right) \mathrm{did}$ 
Table 3

Electrochemical half-wave reduction potentials for compounds showing cathodic reduction in the presence of $\mathrm{CO}_{2}$

\begin{tabular}{lc}
\hline Compound & Reduction \\
\hline $\mathbf{2}$ & $\mathrm{E}_{\mathrm{p} 1 / 2}=-1.65 \mathrm{~V}$ \\
$\mathbf{3}$ & $\mathrm{E}_{\mathrm{p} 1 / 2}=-1.65 \mathrm{~V}$ \\
$\mathbf{4}$ & $\mathrm{E}_{\mathrm{p} 1 / 2}=-1.88 \mathrm{~V}$ \\
$\mathbf{6}$ & $\mathrm{E}_{\mathrm{p} 1 / 2}=-1.70 \mathrm{~V}$ \\
\hline
\end{tabular}

not allow the observation of any chemical-reversibility for 4 . The $E_{p}-E_{p / 2}$ value of 4 is $240 \mathrm{mV}$ at $v=0.2 \mathrm{~V} \mathrm{~s}^{-1}$, diagnostic of a slow charge transfer process with a transfer coefficient $(\alpha)$ of 0.20 [20]. For all of the compounds, no peak was observed in the oxidative region.

For compounds $\mathbf{2 , 3 , 4}$, and $\mathbf{6}$, bulk coulometries were carried out at a controlled potential $150 \mathrm{mV}$ more negative than the potential of the cathodic wave observed by $\mathrm{CV}$. Both coulometries in the absence of or in the presence of $\mathrm{CO}_{2}$ reached completion after the passage of $1 \mathrm{~F}$ per mol of compound through the solution. This confirms therefore the cathodic event is a one-electron process and that no electrocatalytic reduction is taking place. Unfortunately, in both cases, the mono-electronically reduced product undergoes a rapid follow-up chemical reaction which did not allow identification of its structure. The fact that the complexes display a cathodic reduction only in presence of $\mathrm{CO}_{2}$, due to the reduction of a $\mathrm{CO}_{2}$ adduct with the complexes, is a very promising starting point for the development of a new family of $\mathrm{CO}_{2}$ electrocatlaysts. Further investigations will be carried out using ultrafast voltammetry combined with spectroelectrochemistry in order to shed light on the mechanism of reduction and identify the structure of the intermediates.

\section{Conclusions}

The ability to use $\mathrm{CO}_{2}$ as a $\mathrm{C} 1$ synthon for the production of useful chemicals is a longstanding goal of the chemical community. This endeavor still has much room for improvement both in the development of suitable catalysts, but also in the fundamental understanding of how to best activate $\mathrm{CO}_{2}$. A handful of reports are now reporting successful catalytic transformations of $\mathrm{CO}_{2}$ using catalysts that have weak interactions with $\mathrm{CO}_{2}$, which will surely lead to further investigations into this concept. Herein we report the synthesis and characterization of several new $\mathrm{Al}$ and Ga phosphinobenzenethiolates and phosphinophenolates, which were shown to have these weak interactions with $\mathrm{CO}_{2}$. This was exhibited by both the lack of stable adduct formation seen in NMR and FT-IR experiments, along with the cathodic waves observed in $\mathrm{CV}$ experiments upon exposure to $\mathrm{CO}_{2}$. Although none of the electrochemical experiments afforded catalytic transformations of the $\mathrm{CO}_{2}$, we were able show that many of our catalyst designs were capable of activating $\mathrm{CO}_{2}$.

\section{Acknowledgments}

This work was financially supported by the WiSys Technology Foundation (Applied Research Grant 106-Y-06-8000-4), the National Science Foundation (Grant CHE12-13529) to RAK. The Bruker X-ray diffractometer was purchased via a National Science Foundation CRIF:MU award to the University of New Mexico (CHE04-43580), and the NMR spectrometers were upgraded via grants from the NSF (CHE08-40523 and CHE09-46690). Sandia is a multi-mission laboratory managed and oper- 
ated by National Technology and Engineering Solutions of Sandia, LLC., a wholly owned subsidiary of Honeywell International, Inc., for the United States Department of Energy's National Nuclear Security Administration under contract DE-NA0003525. The electrochemical analyses were supported by Nazarbayev University (ORAU grant for Medicinal Electrochemistry) and the Ministry of Education and Science of Kazakhstan. (KL) A partial structure solution for compound 2, the crystal structure for the untargeted complex $\mathrm{LGaMe}\left(\mathrm{C}_{6} \mathrm{~F}_{5}\right.$ ) (where $\mathrm{L}=\mathrm{SC}_{6} \mathrm{H}_{4}-2-\mathrm{P}^{t} \mathrm{Bu}_{2}$ ), all $\mathrm{NMR}$ spectra, and CV scans for compounds 2 and $\mathbf{6}$ can be made available by request to the corresponding author. (BB) CCDC 1546738-1546742 and 1563648 contain the supplementary crystallographic data for complexes $\mathbf{3}, \mathbf{4}, \mathbf{6}-\mathbf{8}$ and the untargeted complex. These data can be obtained free of charge via http://www.ccdc.cam.ac.uk/conts/retrieving/html, or from the Cambridge Crystallographic Data Centre, 12 Union Road, Cambridge CB2 1EZ, UK; fax: +44 1223336 033; or e-mail: E-mail: deposit@ccdc.cam.ac.uk.

\section{References}

[1] (a) J. Klankermayer, S. Wesselbaum, K. Beydoun and W. Leitner, Angew Chem Int Ed 55(26) (2016), 7296-7343; (b) J. Ma, N. Sun, X. Zhang, N. Zhao, F. Xiao, W. Wei and Y. Sun, Catal Today 148(3-4) (2009), 221-231; (c) P. Alagi, R. Ghorpade, Y.J. Choi, U. Patil, I. Kim, J.H. Baik and S.C. Hong, ACS Sustainable Chem Eng 5(5) (2017), 3871-3881; (d) A.-H. Liu, Y.-N. Li and L.-N. He, Pure Appl Chem 84(3) (2012), 581-602.

[2] (a) M. Aresta, C.F. Nobile, V.G. Albano, E. Forni and M. Manassero, J Chem Soc Chem Commun (15) (1975), 636-637; (b) W. Leitner, Coord Chem Rev 155 (1996), 247; (c) D. Walther, M. Ruben and S. Rau, Coord Chem Rev 182(1) (1999), 67-100; (d) D.H. Gibson, Coord Chem Rev 185-186 (1999), 335-355; (e) D.H. Gibson, Chem Rev 96(6) (1996), 2063-2095.

[3] N. von Wolff, G. Lefèvre, J.C. Berthet, P. Thuéry and T. Cantat, ACS Catal. 6(7) (2016), 4526-4535.

[4] I. Andersson, S. Knight, G. Schneider, Y. Lindqvist, T. Lundqvist, C.I. Braenden and G.H. Lorimer, Nature 337(6204) (1989), 229-234.

[5] (a) D.A. Dickie, M.V. Parkes and R.A. Kemp, Angew Chem Int Ed 47(51) (2008), 9955-9957; (b) C.A. Stewart, D.A. Dickie, M.V. Parkes, J.A. Saria and R.A. Kemp, Inorg Chem 49(23) (2010), 11133-11141; (c) D.A. Dickie, E.N. Coker and R.A. Kemp, Inorg Chem 50(22) (2011), 11288-11290; (d) C.A. Stewart, D.A. Dickie, Y. Tang and R.A. Kemp, Inorg Chim Acta 376(1) (2011), 73-79; (e) D.A. Dickie, K.B. Gislason and R.A. Kemp, Inorg Chem 51(2) (2012), 1162-1169; (f) A.M. Felix, B.J. Boro, D.A. Dickie, Y. Tang, J.A. Saria, B. Moasser, C.A. Stewart, B.J. Frost and R.A. Kemp, Main Group Chem 11(1) (2012), 13-29; (g) C.A. Stewart, D.A. Dickie and R.A. Kemp, Inorg Chim Acta 392 (2012), 268-276; (h) C.A. Stewart, D.A. Dickie, B. Moasser and R.A. Kemp, Polyhedron 32(1) (2012), 14-23; (i) B.M. Barry, D.A. Dickie, L.J. Murphy, J.A. Clyburne and R.A. Kemp, Inorg Chem 52(15) (2013), 8312-8314; (j) B.M. Barry, B.W. Stein, C.A. Larsen, M.N. Wirtz, W.E. Geiger, R. Waterman and R.A. Kemp, Inorg Chem 52(17) (2013), 9875-9884; (k) D.A. Dickie, R.P. Ulibarri-Sanchez Iii, P.J. Jarman and R.A. Kemp, Polyhedron 58 (2013), 92-98; (1) D.A. Dickie and R.A. Kemp, Organometallics 33(22) (2014), 6511-6518; (m) D.A. Dickie, M.T. Barker, M.A. Land, K.E. Hughes, J.A. Clyburne and R.A. Kemp, Inorg Chem 54(23) (2015), 11121-1116; (n) R.A. Kemp, D.A. Dickie, E.S. Donovan and B. Barry, Carbon dioxide transformation facilitated by earth abundant metals. WO2015134457A1, 2015; (o) D.A. Dickie, B.E. Chacon, A. Issabekov, K. Lam and R.A. Kemp, Inorg Chim Acta 453 (2016), 42-50.

[6] (a) P. Spies, G. Erker, G. Kehr, K. Bergander, R. Frohlich, S. Grimme and D.W. Stephan, Chem Commun (47) (2007), 5072-5074; (b) D.W. Stephan, Org Biomol Chem 6(9) (2008), 1535-1539; (c) D.W. Stephan, Dalton Trans (17) (2009), 3129-3136.

[7] (a) C.M. Mömming, E. Otten, G. Kehr, R. Fröhlich, S. Grimme, D.W. Stephan and G. Erker, Angew Chem Int Ed 48(36) (2009), 6643-6646; (b) C. Appelt, H. Westenberg, F. Bertini, A.W. Ehlers, J.C. Slootweg, K. Lammertsma and W. Uhl, Angew Chem Int Ed Engl 50(17) (2011), 3925-3928; (c) E. Theuergarten, T. Bannenberg, M.D. Walter, D. Holschumacher, M. Freytag, C.G. Daniliuc, P.G. Jones and M. Tamm, Dalton Trans 43(4) (2014), 1651-1662; (d) D.W. Stephan, Acc Chem Res 48(2) (2015), 306-316; (e) D.W. Stephan, J Am Chem Soc 137(32) (2015), 10018-10032.

[8] S.J. Geier and D.W. Stephan, J Am Chem Soc 131(10) (2009), 3476-3477.

[9] E.S. Donovan, B.M. Barry, C.A. Larsen, M.N. Wirtz, W.E. Geiger and R.A. Kemp, Chem Commun 52(8) (2016), 1685-1688.

[10] (a) F. Buss, P. Mehlmann, C. Muck-Lichtenfeld, K. Bergander and F. Dielmann, J Am Chem Soc 138(6) (2016), 1840-1843; (b) M.A. Courtemanche, M.A. Legare, L. Maron and F.G. Fontaine, J Am Chem Soc 135(25) (2013), 9326-9329. 
[11] M. Bornand, S. Torker and P. Chen, Organometallics 26(14) (2007), 3585-3596.

[12] (a) G. Jarre, S. Heyer, E. Memmel, T. Meinhardt and A. Krueger, Beilstein J Org Chem 10 (2014), 2729-2737; (b) D.W. Stephan, J.C. Stewart, F. Guérin, S. Courtenay, J. Kickham, E. Hollink, C. Beddie, A. Hoskin, T. Graham, P. Wei, R.E.v.H. Spence, W. Xu, L. Koch, X. Gao and D.G. Harrison, Organometallics 22(9) (2003), 1937-1947; (c) N. Fechler, T.-P. Fellinger and M. Antonietti, Adv Mater 25(1) (2013), 75-79.

[13] G. Gritzner and J. Kůta, Pure Appl Chem 56(4) (1984), 461-466.

[14] R.R. Gagne, C.A. Koval and G.C. Lisensky, Inorg Chem 19(9) (1980), 2854-2855.

[15] APEX2, Bruker AXS, Inc.: 2007.

[16] G.M. Sheldrick, Acta Crystallogr, Sect A: Found Crystallogr 64(1) (2008), 112-122.

[17] K. Brandenburg, Diamond 3.2i, Crystal Impact GbR: 2012.

[18] J. Klosin, G.R. Roof, E.Y.X. Chen and K.A. Abboud, Organometallics 19(23) (2000), 4684-4686.

[19] In practical terms, electrochemical reversibility (also termed Nernstian behavior) refers to the speed of charge-transfer in a redox reaction, whereas chemical reversibility refers to follow-up reactions that accompany the charge-transfer process. For an introductory discussion of these terms, see A.J. Bard and L.N. Faulkner, Electrochemical Methods, John Wiley \& Sons, New York, 2001, 2nd Ed., pp 35-38 and pp 44-49).

[20] See ref. 18, page 236 . 